\section{Evaluation the FLAIR Sensitivity and DWI Post-inject in Comparison with Delayed Enhancement T1w for Better Detection of Active MS Lesions}

\author{
Hoseinipourasl M. ${ }^{1}$, Zandkarimi M. ${ }^{1}$, Abdolmohammadi \\ J. ${ }^{2 *}$, Sharifi K. ${ }^{3}$, Miraki S. ${ }^{4}$
}

\begin{abstract}
Background: Multiple sclerosis (MS) is a chronic, typically progressive and most common autoimmune disease which damaged the central nervous system. According to the reports in 2008, this disorder has affected 2 and 2.5 million people globally. While the reason is not clear, proposed causes for this include immunologic, environmental, infectious and genetic factors, and sexuality. MS can cause many symptoms, including blurred vision, loss of balance, poor coordination, slurred speech, tremors, numbness, extreme fatigue, problems with memory and concentration, paralysis, blindness, and more. There are four distinguished illness fields in MS: relapsing-remitting MS (RRMS), primary-progressive MS (PPMS), secondaryprogressive MS (SPMS), and progressive-relapsing. MRI is a great tool to identify the asymptomatic distribution of lesions in space and time.

Materials and Methods: 32 patients with MS plaques were evaluated by FLAIR and DWI pre- and post-Gadolinium injection compared with 15minutes delay T1w SE.
\end{abstract}

Results: FLAIR post-inject had significantly better detection of the number and signal intensity of active MS lesions. DWI and ADC images detected active plaques different from non-active lesions without contrast.

Conclusion: The result of this study showed that FLAIR post-inject had the highest sensitivity in detection of active MS lesions due to the CSF signal suppression in FLAIR, thus offering enough TR time recovery in active enhanced plaques.

\section{Keywords}

Multiple Sclerosis, MRI, FLAIR, Contrast media

\section{Introduction}

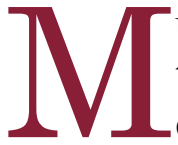
ultiple sclerosis is the most common autoimmune disorder through which covers of nerve cells in the brain and spinal cord are damaged [1]. The disease typically starts up between the ages of 20 and 50 and is more common in women (twice as common in women as in men) [2]. In multiple sclerosis, the body's immune system wrongly assault myelin (a protective cover around our nerves), then, it causes inflammation and damage (demyelination) [3]; generally, these lesions (resulting scared tissue is called a lesion) are active or inactive which active lesions forming or expanding can cause a wide variety
${ }^{1}$ Department of Radiology, BSc of Radiology,

School of Paramedical

Sciences, Kurdistan

University of Medical

Sciences, Sanandaj, Iran

${ }^{2}$ Department of Radiol-

ogy, Faculty of Paramedi-

cal Sciences, Kurdistan

University of Medical

Sciences, Sanandaj, Iran

${ }^{3}$ Professor Assistant of

Radiology, Kurdistan

Department of Radiol-

ogy, Faculty of Medical

Sciences, Kurdistan

University of Medical Sci-

ences, Sanandaj, Iran

${ }^{4} \mathrm{MSc}$ of Anatomy, Sha-

hid Chamran MRI center,

Kurdistan University of

Medical Sciences, San-

andaj, Iran

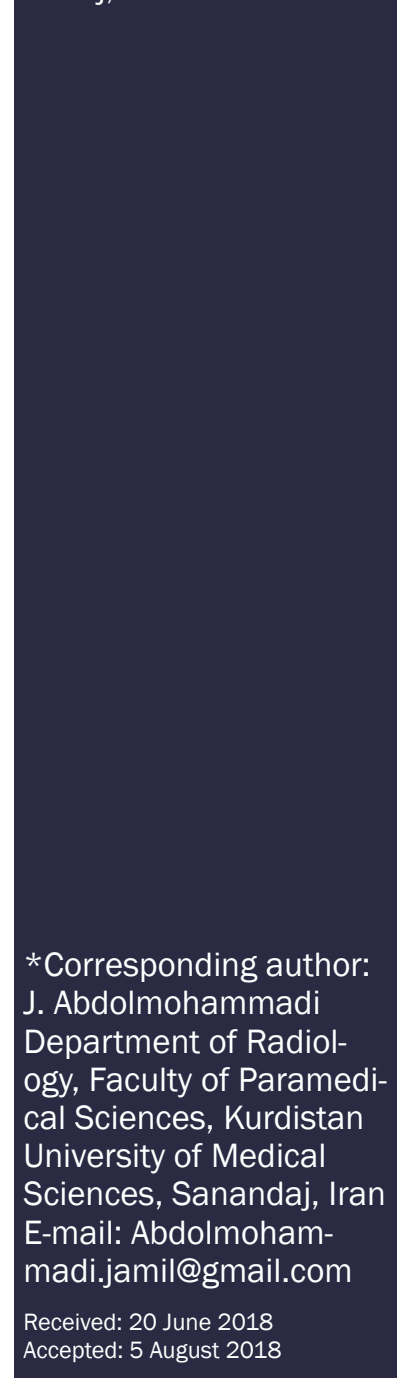


of symptoms, according to their location and size, however, inactive ones do not cause any symptoms [4].

Numerous signs are caused by MS that include: loss of balance, blurred vision, slurred speech, poor coordination, extreme fatigue, tremors, numbness, memory and concentration problems, etc. These obstacles may appear and leave or stay and get worse over time [5]. There are four distinguished illness fields in MS: relapsing-remitting MS (RRMS), primary-progressive MS (PPMS), secondaryprogressive MS (SPMS), and progressiverelapsing. They could be benign, equivalent, or intensive [6]. While the reason is not clear, proposed causes for this include immunologic factors, environmental factors, infectious factors, genetic factors, and sexuality [7].

MS is diagnosed by various tests such as MRI and it is a great tool to identify the asymptomatic distribution of lesions in mentioned dissemination [9]. The methods of MS distinction are according to revealing the distribution of white matter lesions [9]. There are lots of important roles of MRI that they could be mentioned; for instance one of the most significant uses of MRI in the diagnostic work-up of suspicious MS patients is to perform further diagnoses apparent on MRI such as spinal stenosis and most brain tumors [10].

The schema and progress of MRI lesions, in the suitable clinical context, have made MRI abnormalities precious criteria for the early diagnosis of MS [11]. The sensitivity of diagnosing MS during the first year is $94 \%$ after a single attack, in the specificity of $83 \%$ [12]. The other significant role of MRI is to early diagnose MS for clinically isolated syndrome (CIS) patients using the international panel (IP) diagnostic criteria including MRI for dissemination in space (DIS) and time (DIT) [13]. Raising the Authorizing a new MRI lesion to the replacement for a clinical attack doubles up the number of CIS patients who can be recognized as possessing MS after one year of symptom beginning.
The MRI data needed to support the diagnosis varies, rely on the potency of the clinical detections [14]. Specific lesions noticing MS include ovoid lesions, Dawson fingers, asymptomatic spinal cord lesions, and corpus callosum. Nevertheless, another white matter disease can have an equivalent appearance on an MRI [15].

Fluid-attenuated inversion recovery (FLAIR) is a proper sequence to distinguish MS lesions in the periventricular area that is a common region for multiple sclerosis sores that they can be managed by suppressing the signal from CSF yet sustaining heavily T2 weighting [16]. Aforementioned sequence is excellent at discovering cortical/juxtacortical injuries. Consequently, FLAIR is a suitable MR sequence on clinical scanners when MS has been elevated as a feasible clinical research. Distinguishing lesions with substandard quality by PD and $\mathrm{T} 2$ weighting in the posterior fossa and spinal cord where these sequences are prosperous is the disadvantage. [17, 18].

Five minutes before T1 weighted imaging, a gadolinium is given intravenously to distinguish the blood-brain barrier (BBB) disruption in collaboration with active inflammation. New lesions enhanced after injection are valuable markers for controlling disease activity which endure for a month on average. These lesions perform a significant function in designating diffusion in time within the new diagnostic rules [19]. An improvement in active lesions discovery can be earned by a triple dose of gadolinium or combination with magnetization transfer imaging, however, are not needed in clinical usage [20].

Despite normal MRI images, a few patients are believed to still suffer from MS. One of the most important matters that it should be mentioned here is that whether such patients lack MS plaques or MRI scans are not sufficiently optimized to detect MS plaques. However, investigating for the proper MRI sequences in detecting MS plaques is the main object of this study. 


\section{Materials and Methods}

In this cross-sectional study, 32 sick people (male $=10$, female $=22$, range $18-38$ yearold, average of age: 27 year-old) be subjected to MRI assessment between May 2014, and February 2015 at Chamran Imaging Center, Sanandaj, Iran. MRI imaging was performed using a Siemens, Avanto, 1.5 Tesla system equipped with eight-channel head quadrature coil.

First, images without contrast were obtained, including T2w-FSE, T1w-FSE, FLAIR, SEEPI (DWI) sequences (see Table 1). Then, 0.1 $\mathrm{mmol} / \mathrm{kg}$ contrast media (Gadolinium-based)

Table 1: Sequences parameters.

\begin{tabular}{llccccccc} 
Parameters Sequence & \multicolumn{1}{c}{ Plane } & $\begin{array}{c}\text { TR } \\
(\mathbf{m})\end{array}$ & $\begin{array}{c}\text { TE } \\
(\mathbf{m s})\end{array}$ & NEX & Matrix size & $\begin{array}{c}\text { FOV } \\
(\mathbf{c m})\end{array}$ & $\begin{array}{c}\text { Slice thick- } \\
\text { ness }(\mathbf{m m})\end{array}$ & $\begin{array}{c}\text { Gap } \\
(\mathbf{m m})\end{array}$ \\
\hline T2W-FSE & Axial and Coronal & 3700 & 113 & 1 & $256 \times 320$ & 24 & 5 & 1 \\
\hline T1W-FSE & Axial and Sagittal & 410 & 9 & 1 & $256 \times 320$ & 24 & 5 & 1 \\
\hline $\begin{array}{l}\text { FLAIR Pre- and post- } \\
\text { inject }\end{array}$ & Axial & 8000 & 90 & 2 & $256 \times 320$ & 24 & 5 & 1 \\
\hline SE-EPI (DWI) & Axial & 3100 & 103 & 4 & $128 \times 128$ & 26 & 5 & 1 \\
\hline T1W-FSE Post inject & $\begin{array}{l}\text { Axial Coronal } \\
\text { Sagittal }\end{array}$ & 410 & 9 & 1 & $256 \times 320$ & 24 & 5 & 1
\end{tabular}

was injected for each patient; finally, FLAIR and T1w-FSE images (in three planes) were obtained again with 30-minute delay after injection. The related images to our study were evaluated by two radiologists, and the number of the observed active MS plaques in these sequences was noted. The comparison of observed MS plaques in three different MRI pulse sequences was provided by ANOVA analysis test $(P<0.05)$, and the amount of agreement between two radiologists has been made with Kappa statistic.

\section{Results}

This study was performed on 32 patients (male:10; female: 22); 110 MS plaques observed of which of them have been active, which FLAIR post-injection sequences showed that they have the most sensitivity followed by T1W-post-injection; DWI and ADC map have the most sensitivity, respectively. FLAIR pre-injection demonstrated 94 inactive MS plaques (see Tables 2 and 3). These images were evaluated by two radiologists, and the results were analyzed by SPSS software version 20 by ANOVA analytical test.

The results revealed that, in FLAIR postinjection images, more acute MS plaques at the supra-tentorial area have been observed than in the T1W-TSE post-injection, which is a gold standard sequence. DWI sequences and ADC map displayed active plaques and even some of the inactive plaques, which had less specificity compared to the two above-mentioned sequences $(p<0.05)$. (see Table 4$)$. Also, the results revealed that FLAIR post-inject has less Positive Predictive Value (PPV), than

Table 2: Results of Article about Active MS Plaques.

\begin{tabular}{lcc} 
& $\begin{array}{c}\text { Infra-tento- } \\
\text { rial Plaques }\end{array}$ & $\begin{array}{c}\text { Supra-tento- } \\
\text { rial Plaques }\end{array}$ \\
\hline $\begin{array}{l}\text { FLAIR Post-Inject } \\
\text { (Active plaques) }\end{array}$ & 1 & 16 \\
\hline DWI (Active plaques) & 1 & 11 \\
\hline $\begin{array}{l}\text { ADC (Active } \\
\text { plaques) }\end{array}$ & 1 & 13 \\
\hline $\begin{array}{l}\text { T1w-TSE Post-Inject } \\
\text { (Active plaques) }\end{array}$ & 2 & 14
\end{tabular}


Table 3: Other Results About Non-Active MS Plaques.

\begin{tabular}{lcc} 
& $\begin{array}{c}\text { Infra-tento- } \\
\text { rial Plaques }\end{array}$ & $\begin{array}{c}\text { Supra-tento- } \\
\text { rial Plaques }\end{array}$ \\
\hline $\begin{array}{l}\text { FLAIR Pre-Inject } \\
\text { (Non-Active plaques) }\end{array}$ & 5 & 94 \\
\hline $\begin{array}{l}\text { T1w TSE Pre-Inject } \\
\text { (Non-Active plaques) }\end{array}$ & 3 & 69 \\
\hline $\begin{array}{l}\text { DWI (Non-Active } \\
\text { plaques) }\end{array}$ & 2 & 73 \\
\hline $\begin{array}{l}\text { ADC (Non-Active } \\
\text { plaques) }\end{array}$ & 2 & 77
\end{tabular}

T1w gold standard and DWI, but it has more Negative Predictive Value (NPV) in comparison with T1w gold standard (see Table 5).

There is no significant difference between FLAIR post-inject and T1w-TSE post-inject as well as between T1w-TSE post-inject and ADC. Because the important level between these two evaluations is more than 0.05 , other cases have considerable statistically dissension. The most differences and strong sequences are related to the FLAIR post-inject because of the greater difference average in comparison with other sequences.

\section{Discussion}

Multiple sclerosis (MS) as a central nervous system disease is determined by a collection of pathophysiological activities containing inflammation, demyelination, and axonal loss.
MRI is the primary imaging modality to diagnose MS and track up the plaques and it has a high sensitivity to detect the plaques. Contrast enhancement in MS plaques indicates activation and its superior application in the clinical assessment. Several sequences of MRI, like $\mathrm{T}_{1}$ (with contrast) - and $\mathrm{T}_{2} \mathrm{~W}$ SE, MT-prepared imaging, FLAIR, STIR, and DWI, applied for the MS detection.

The substantial problem that we have been faced recently is showing the tiny MS plaques for patients with suspected MS. Although large plaques may be obvious in all images [20]. This study has proved that FLAIR sequences had acceptable accuracy in detecting MS lesions along with $\mathrm{T} 1 \mathrm{w}$ post-inject for showing the active MS lesions placed in the subcortical and infra-tentorial areas. Thus, studies indicate that it seems not to be enough to assess MS plaque and act alone because FLAIR has imaging advantages including high contrast to noise as well as post-inject [22]. In addition to the FLAIR sequence, other sequences such as $\mathrm{PD}$ and $\mathrm{T} 2$ can be helpful, especially in presaturation fat sat pulses usage [23]. However, new research has shown that the 3D doubleinversion recovery sequence has high diagnostic accuracy in displaying of MS plaques especially in juxta-cortical. Finally, it seems that, in order to better detect active brain MS lesions, it is necessary to use some related sensitive sequences; otherwise, some tiny active MS lesions may be missed.

Table 4: Statistical analyze of this study. The findings reveal significant differences between all the studied sequences in the supra stage $(F 3,28=14.98$; Sig=0.000). Thus, the LSD post-hoc test is used for studying the differentiation between sequences.

ANOVA

\begin{tabular}{|c|c|c|c|c|c|}
\hline Score2 & Sum of Squares & df & Mean Square & $\mathbf{F}$ & Sig \\
\hline Between Groups & 413.594 & 3 & 137.865 & \multirow{3}{*}{14.984} & 0.000 \\
\hline Within Groups & 257.625 & 28 & 9.201 & & \\
\hline Total & 671.219 & 31 & & & \\
\hline
\end{tabular}


Table 5: Multiple Comparisons analyze of this study.

\section{Multiple Comparisons}

\begin{tabular}{|c|c|c|c|c|c|c|}
\hline \multicolumn{7}{|l|}{ Score 2 LSD } \\
\hline \multirow[b]{2}{*}{ (I) section } & \multirow[b]{2}{*}{ (J) section } & \multirow{2}{*}{$\begin{array}{l}\text { Mean Dif- } \\
\text { ference } \\
(\mathrm{I}-\mathrm{J})\end{array}$} & \multirow{2}{*}{$\begin{array}{l}\text { Std. } \\
\text { Error }\end{array}$} & \multirow[b]{2}{*}{ Sig } & \multicolumn{2}{|c|}{ 95\% Confidence Interval } \\
\hline & & & & & $\begin{array}{l}\text { Lower } \\
\text { Bound }\end{array}$ & $\begin{array}{l}\text { Upper } \\
\text { Bound }\end{array}$ \\
\hline \multirow[t]{3}{*}{ FLAIR Post Inject } & DWI & $9.875^{*}$ & 1.517 & 0.001 & 6.77 & 12.98 \\
\hline & ADC & $3.875^{*}$ & 1.517 & 0.016 & 0.77 & 6.98 \\
\hline & T1w-TSE Post-Inject & 2.875 & 1.517 & 0.068 & -0.23 & 5.98 \\
\hline \multirow[t]{3}{*}{ DWI } & FLAIR Post-Inject & $-9.875^{*}$ & 1.517 & 0.001 & -12.98 & -6.77 \\
\hline & ADC & $-6.000^{*}$ & 1.517 & 0.001 & -9.11 & -2.89 \\
\hline & T1w-TSE Post- Inject & $-7.000^{*}$ & 1.517 & 0.001 & -10.11 & -3.89 \\
\hline \multirow[t]{3}{*}{ ADC } & FLAIR Post- Inject & $-3.875^{\star}$ & 1.517 & 0.016 & -6.98 & -0.77 \\
\hline & DWI & $6.000^{*}$ & 1.517 & 0.001 & 2.89 & 9.11 \\
\hline & T1w-TSE Post- Inject & -1.000 & 1.517 & 0.515 & -4.11 & 2.11 \\
\hline \multirow[t]{3}{*}{ T1w-TSE Post Inject } & FLAIR Post- Inject & -2.875 & 1.517 & 0.068 & -5.98 & 0.23 \\
\hline & DWI & $7.000^{*}$ & 1.517 & 0.001 & 3.89 & 10.11 \\
\hline & ADC & 1.000 & 1.517 & 0.515 & -2.11 & 4.11 \\
\hline
\end{tabular}

${ }^{*}$ The mean difference is significant at the 0.05 level.

There were some restrictions on the message data status on the active plaques or not to use the double dose because the patient may have a special situation such as kidney problems and the treatment expenditure. Also, a DIR sequence is not used due to shortages in the treatment center.

In this study, we investigated the relative contribution of several MRI criteria derived from Gadolinium-enhanced post-contrast FLAIR, DWI, and gadolinium-enhanced T1-weighted images in order to detect multiple sclerosis in MS patients. On FLAIR images, signals from the CSF are nulled, thus increasing contrast between lesions and adjacent CSF. Our results indicate that a FLAIR sequence was better at lesion visualization than the DWI, T1w, sequences tested herein (Figures 1 and 2).

There is another important factor in these discoveries that the number of active plaques is found, and it always seems to be more. It means high specificity and sensitivity in the messages in comparison with $\mathrm{T} 1 \mathrm{w}$ post-injection numbers of real plaques.

To identify wounds, diffusion tensor imaging is really sensitive where myelin disruption has caused an enhancment in apparent diffusion coefficient (ADC) and diminished fractional anisotropy (FA). Nevertheless, the aforementioned process does not have specificity because it reveals each demyelination, gliosis, inflammation, axonal contraction, and axonal loss. In this investigation, in some cases, the DWI sequence was not able to detect active plaques and as well as the DWI sequence detecting some non-active plaques as active (Figures 3 and 4). This survey does not have such the similar fulfillment. 


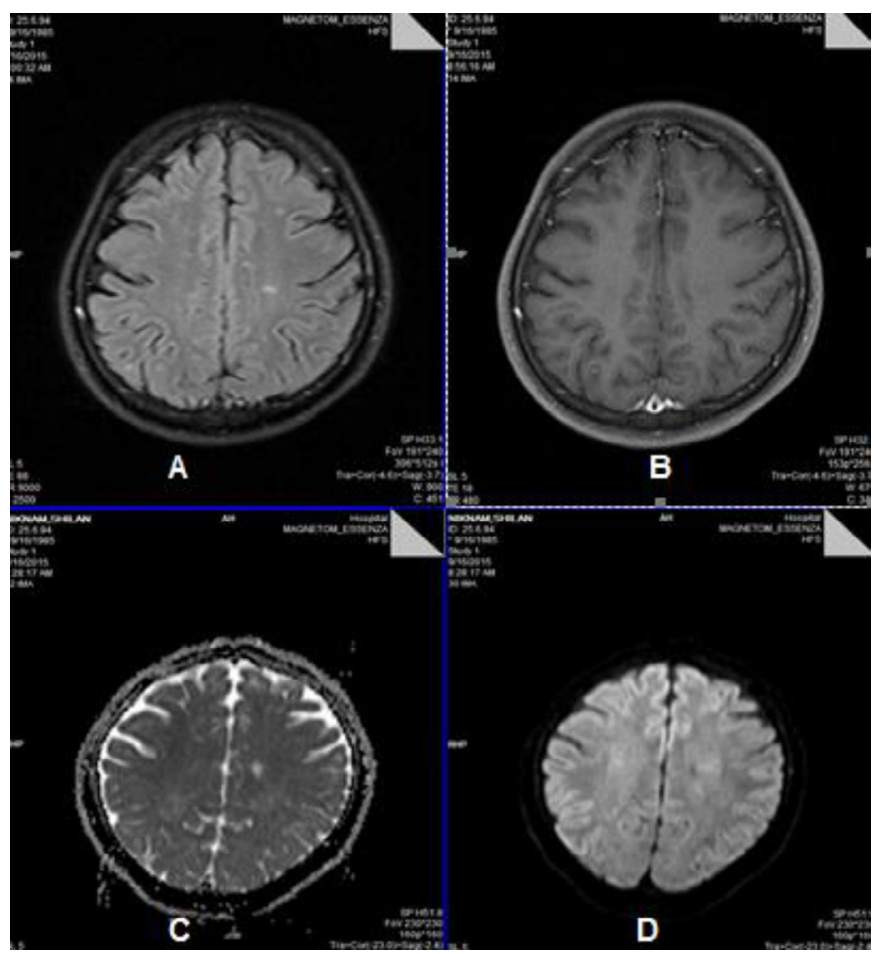

Figure 1: A tiny active MS plaque in subcortical white matter in parietal lobe area with four MRI pulse sequences: A) FLAIR post-inject (high signal); B) T1w post inject (iso intense); C) ADC map (high signal); D) DWI with b value 1000 (iso intense to high signal, restricted).
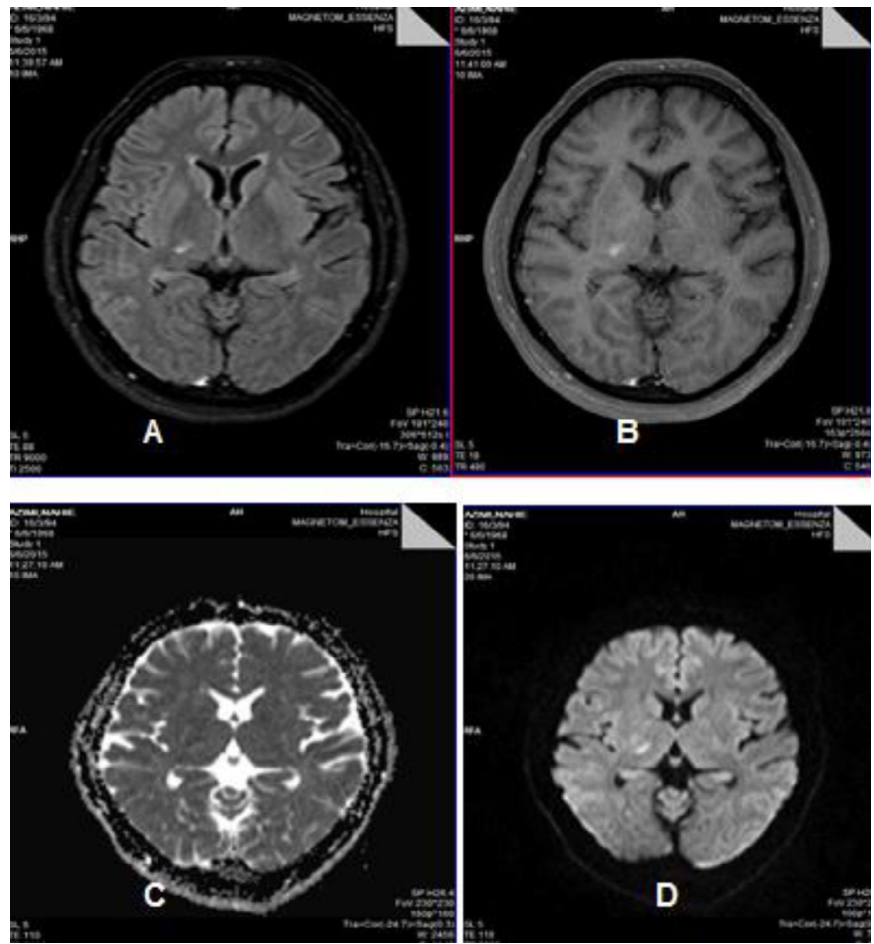

Figure 2: An Active MS plaque in periventricular area with four MRI pulse sequences: A) FLAIR post inject (high signal); B) T1w post inject (high signal); C) ADC map (low signal); D) DWI with b value 1000 (high signal). 

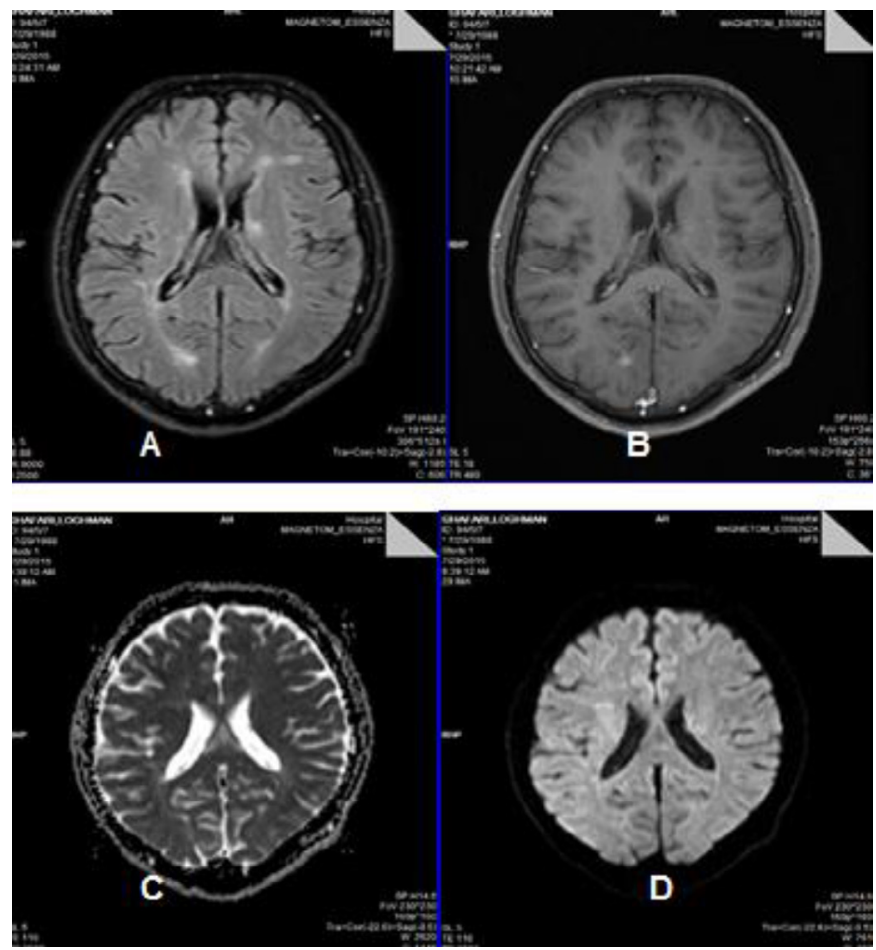

Figure 3: An active MS plaque in occipital lobe with four MRI pulse sequence: A) FLAIR postinject (high signal); B) T1w post-inject (iso intense to high signal); C) ADC map (low signal); D) DWI with b value 1000 (low signal).
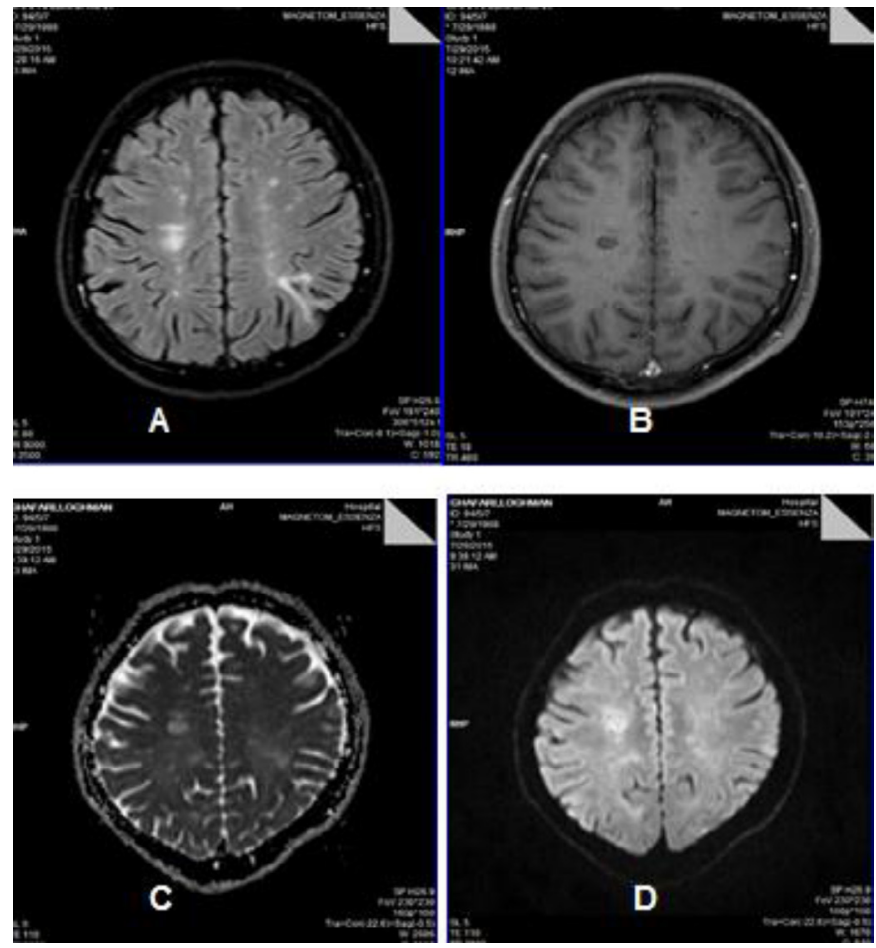

Figure 4: Non-active MS plaque in subcortical white matter in parietal lobe area with four MRI pulse sequences: A) Flair pre-inject (high signal); B) T1w post-inject (low signal); C) ADC map (high signal); D) DWI with b value 1000 (high signal). 


\section{Conclusion}

In some cases, however, the patient has symptoms in which MS is suspected, MRI images are regular, or maybe the patient has symptoms related to a specific area of the brain, MRI images do not show the MS plaques in the given area. Therefore, in this situation, we cannot surely conclude that the patient doesn't have MS plaques because, in some cases, it is poor in the optimization of MRI pulse sequences. Thus, in this investigation, we have some different MRI pulse sequences evaluated to asses which of them is the most efficient so as to detect MS plaques in the brain. For this reason, we obtained additional MRI pulse sequences rather than doing only routine brain MRI.

\section{Acknowledgment}

This study was fiscally backed by the Vice Chancellor for Research and Technological of Kurdistan University of Medical Sciences. We are thankful to research radiographer Vafa samandnejad for the technical aid for carrying out the experiments. Thanks, loads to our colleague Dr. Reza Yasini Farid, who brought the statistical analysis that greatly aided the researches

\section{Conflict of Interest}

There is no contradictory of zeal to be conveyed.

\section{References}

1. Darlington PJ, Touil T, Doucet JS, Gaucher D, Zeidan J, Gauchat D, et al. Diminished Th17 (not Th1) responses underlie multiple sclerosis disease abrogation after hematopoietic stem cell transplantation. Ann Neurol. 2013;73:341-54. doi: 10.1002/ ana.23784. PubMed PMID: 23463494.

2. T Jock Murray M. Multiple sclerosis: the history of a disease. Ne York: Demos medical publishing; 2004.

3. Lutterotti A, Yousef S, Sputtek A, Sturner $\mathrm{KH}$, Stellmann JP, Breiden $\mathrm{P}$, et al. Antigenspecific tolerance by autologous myelin pep- tide-coupled cells: a phase 1 trial in multiple sclerosis. Sci Transl Med. 2013;5:188ra75. doi: $\quad$ 10.1126/scitransImed.3006168. PubMed PMID: 23740901; PubMed Central PMCID: PMC3973034.

4. Genain CP, Cannella B, Hauser SL, Raine CS. Identification of autoantibodies associated with myelin damage in multiple sclerosis. Nat Med. 1999;5:170-5. doi: 10.1038/5532. PubMed PMID: 9930864.

5. Zajicek J, Fox P, Sanders H, Wright D, Vickery J, Nunn A, et al. Cannabinoids for treatment of spasticity and other symptoms related to multiple sclerosis (CAMS study): multicentre randomised placebo-controlled trial. Lancet. 2003;362:1517-26. doi: 10.1016/ S0140-6736(03)14738-1.

6. Bove R, Secor E, Healy BC, Musallam A, Vaughan T, Glanz Bl, et al. Evaluation of an online platform for multiple sclerosis research: patient description, validation of severity scale, and exploration of $\mathrm{BMI}$ effects on disease course. PLOS One. 2013;8:e59707. doi: 10.1371/journal. pone.0059707. PubMed PMID: 23527256; PubMed Central PMCID: PMC3603866.

7. Rossi S, Motta C, Studer V, Barbieri F, Buttari $F$, Bergami $A$, et al. Tumor necrosis factor is elevated in progressive multiple sclerosis and causes excitotoxic neurodegeneration. Mult Scler. 2014;20:304-12. doi: $10.1177 / 1352458513498128$. PubMed PMID: 23886826.

8. Krupp LB, Tardieu M, Amato MP, Banwell B, Chitnis T, Dale RC, et al. International Pediatric Multiple Sclerosis Study Group criteria for pediatric multiple sclerosis and immunemediated central nervous system demyelinating disorders: revisions to the 2007 definitions. Mult Scler. 2013;19:1261-7. doi: 10.1177/1352458513484547. PubMed PMID: 23572237.

9. Magliozzi R, Serafini B, Rosicarelli B, Chiappetta $G$, Veroni $C$, Reynolds $R$, et al. B-cell enrichment and Epstein-Barr virus infection in inflammatory cortical lesions in secondary progressive multiple sclerosis. J Neu- 
ropathol Exp Neurol. 2013;72:29-41. doi: 10.1097/NEN.0b013e31827bfc62. PubMed PMID: 23242282.

10. Graham D, Huang S, Choi J, Rudin S, Yu D, Jenkins B, et al. ATX-MS-1467 reduces MRI lesions and prevents disease progression in a humanized mouse model of multiple sclerosis. MULTIPLE SCLEROSIS JOURNAL. 2014;20:235.

11. Huang S, Choi J-K, Gray A, Yu D, Jenkins B, Mandeville J, et al. ONO-4641 (Ceralifimod) Reduces MRI Lesions and Prevents Disease Progression in an Animal Model of Multiple Sclerosis (P1. 219). Neurology. 2014;82:P1. 219.

12. Giorgio A, Stromillo ML, Bartolozzi ML, Rossi F, Battaglini M, De Leucio A, et al. Relevance of hypointense brain MRI lesions for long-term worsening of clinical disability in relapsing multiple sclerosis. Mult Scler. 2014;20:214-9. doi: 10.1177/1352458513494490. PubMed PMID: 23877971.

13. Coles AJ, Twyman CL, Arnold DL, Cohen JA, Confavreux C, Fox EJ, et al. Alemtuzumab for patients with relapsing multiple sclerosis after disease-modifying therapy: a randomised controlled phase 3 trial. Lancet. 2012;380:1829-39. doi: 10.1016/ S0140-6736(12)61768-1. PubMed PMID: 23122650.

14. Filippi M, Rocca MA, Ciccarelli 0, De Stefano N, Evangelou N, Kappos L, et al. MRI criteria for the diagnosis of multiple sclerosis: MAGNIMS consensus guidelines. Lancet Neurol. 2016;15:292-303. doi: 10.1016/S1474-4422(15)00393-2. PubMed PMID: 26822746; PubMed Central PMCID: PMC4760851.

15. Wens I, Dalgas U, Stenager E, Eijnde B0. Risk factors related to cardiovascular diseases and the metabolic syndrome in multiple sclerosis-a systematic review. Multiple Sclerosis Journal. 2013;19:1556-64. doi: $10.1177 / 1352458513504252$.

16. Kolber P, Montag S, Fleischer V, Luessi F, Wilting J, Gawehn J, et al. Identification of cortical lesions using DIR and FLAIR in early stages of multiple sclerosis. $J$ Neurol. 2015;262:1473-82. doi: 10.1007/s00415015-7724-5. PubMed PMID: 25862481.

17. Schmidt $P$, Gaser C, Arsic M, Buck $D$, Forschler A, Berthele A, et al. An automated tool for detection of FLAIR-hyperintense white-matter lesions in Multiple Sclerosis. Neuroimage. 2012;59:3774-83. doi: $\quad$ 10.1016/j.neuroimage.2011.11.032. PubMed PMID: 22119648.

18. Patzig $M$, Burke $M$, Bruckmann $H$, Fesl G. Comparison of 3D cube FLAIR with 2D FLAIR for multiple sclerosis imaging at 3 Tesla. Rofo. 2014;186:484-8. doi: 10.1055/s-0033-1355896. PubMed PMID: 24347360.

19. Absinta $M$, Vuolo $L$, Rao $A$, Nair G, Sati P, Cortese IC, et al. Gadoliniumbased MRI characterization of leptomeningeal inflammation in multiple sclerosis. Neurology. 2015;85:18-28. doi: 10.1212/ WNL.0000000000001587. PubMed PMID: 25888557; PubMed Central PMCID: PMC4501940.

20. Tourdias T, Roggerone S, Filippi M, Kanagaki M, Rovaris M, Miller $\mathrm{DH}$, et al. Assessment of Disease Activity in Multiple Sclerosis Phenotypes with Combined Gadolinium-and Superparamagnetic Iron Oxide-enhanced MR Imaging. Radio/ogy. 2012;264:225-33. doi: 10.1148/radiol.12111416.

21. Lazeron RH, Langdon DW, Filippi $M$, van Waesberghe $\mathrm{JH}$, Stevenson VL, Boringa JB, et al. Neuropsychological impairment in multiple sclerosis patients: the role of (juxta)cortical lesion on FLAIR. Mult Scler. 2000;6:280-5. doi: 10.1177/135245850000600410. PubMed PMID: 10962549.

22. Castillo MS, Davis FG, Surawicz T, Bruner JM, Bigner S, Coons S, et al. Consistency of primary brain tumor diagnoses and codes in cancer surveillance systems. Neuroepidemiology. 2004;23:85-93. doi: 10.1159/000073980. PubMed PMID: 
14739573.

23. Gawne-Cain M, O'riordan J, Thompson A, Moseley I, Miller D. Multiple sclerosis lesion detection in the brain: a comparison of fast fluid-attenuated inversion recovery and conventional T2-weighted dual spin echo. Neurology. 1997;49:364-70. doi: 10.1212/ wnl.49.2.364 . 